\title{
Multiparametric MRI changes persist beyond recovery in concussed adolescent hockey players OPEN
}

Kathryn Y. Manning, MSc

Amy Schranz, BMSc Robert Bartha, PhD

Gregory A. Dekaban, $\mathrm{PhD}$

Christy Barreira, BSc

Arthur Brown, PhD

Lisa Fischer, MD

Kevin Asem, MD

Timothy J. Doherty, MD, $\mathrm{PhD}$

Douglas D. Fraser, MD, $\mathrm{PhD}$

Jeff Holmes, PhD

Ravi S. Menon, PhD

Correspondence to

Dr. Menon:

rmenon@robarts.ca
Supplemental data at Neurology.org

\section{ABSTRACT}

Objective: To determine whether multiparametric MRI data can provide insight into the acute and long-lasting neuronal sequelae after a concussion in adolescent athletes.

Methods: Players were recruited from Bantam hockey leagues in which body checking is first introduced (male, age 11-14 years). Clinical measures, diffusion metrics, resting-state network and region-to-region functional connectivity patterns, and magnetic resonance spectroscopy absolute metabolite concentrations were analyzed from an independent, age-matched control group of hockey players $(n=26)$ and longitudinally in concussed athletes within 24 to 72 hours $(n=17)$ and 3 months $(n=14)$ after a diagnosed concussion.

Results: There were diffusion abnormalities within multiple white matter tracts, functional hyperconnectivity, and decreases in choline 3 months after concussion. Tract-specific spatial statistics revealed a large region along the superior longitudinal fasciculus with the largest decreases in diffusivity measures, which significantly correlated with clinical deficits. This region also spatially intersected with probabilistic tracts connecting cortical regions where we found acute functional connectivity changes. Hyperconnectivity patterns at 3 months after concussion were present only in players with relatively less severe clinical outcomes, higher choline concentrations, and diffusivity indicative of relatively less axonal disruption.

Conclusions: Changes persisted well after players' clinical scores had returned to normal and they had been cleared to return to play. Ongoing white matter maturation may make adolescent athletes particularly vulnerable to brain injury, and they may require extended recovery periods. The consequences of early brain injury for ongoing brain development and risk of more serious conditions such as second impact syndrome or neural degenerative processes need to be elucidated. Neurology ${ }^{\circledR}$ 2017;89:2157-2166

\section{GLOSSARY}

$\mathbf{A D}=$ axial diffusivity; $\mathbf{C S T}=$ corticospinal tract; $\mathbf{D M N}=$ default mode network; $\mathbf{D T I}=$ diffusion tensor imaging; $\mathbf{F A}=$ fractional anisotropy; $\mathbf{M D}=$ mean diffusivity; $\mathbf{M R S}=$ magnetic resonance spectroscopy; $\mathbf{R D}=$ radial diffusivity; $\mathbf{R O I}=$ region of interest; $\mathbf{R S}$ = resting-state; $\mathbf{R S N}$ = resting-state network; SLF = superior longitudinal fasciculus.

Concussion remains a critical health problem, and the developing brain may be uniquely susceptible. ${ }^{1}$ In Canada, hockey-related concussions account for nearly half of all sports teamrelated concussions and disproportionately affect adolescents. ${ }^{2}$ The neuronal mechanisms that underlie concussion remain poorly understood. A complex cascade of structural, functional, and metabolic changes are secondary to rotational acceleration of the brain. ${ }^{3}$ MRI studies have reported neuronal vulnerabilities that persist after athletes have been cleared to return to play. ${ }^{4-7}$ Without full recovery, players may have increased susceptibility to injury and may be at risk of serious conditions such as second impact syndrome. ${ }^{8}$ Therefore, it is essential to understand the

\footnotetext{
From the Department of Medical Biophysics (K.Y.M., A.S., R.B., R.S.M.), Department of Microbiology and Immunology (G.A.D., C.B.), Department of Anatomy and Cell Biology (A.B), Department of Physical Medicine and Rehabilitation (T.J.D.), and School of Occupational Therapy (J.H.), University of Western Ontario; Centre for Functional and Metabolic Mapping (K.Y.M., R.B., R.S.M.) and Molecular Medicine (G.A.D., C.B., A.B.), Robarts Research Institute; Primary Care Sport Medicine (L.F., K.A.), Family Medicine, Fowler Kennedy Sport Medicine; and Paediatrics Critical Care Medicine (D.D.F.), London Health Sciences Centre, London, Ontario, Canada.

Go to Neurology.org for full disclosures. Funding information and disclosures deemed relevant by the authors, if any, are provided at the end of the article. The Article Processing charge was funded by Canadian Institutes of Health Research Foundation Grant 353372 to R.S. Menon.

This is an open access article distributed under the terms of the Creative Commons Attribution-NonCommercial-NoDerivatives License 4.0 (CC BY-NC-ND), which permits downloading and sharing the work provided it is properly cited. The work cannot be changed in any way or used commercially without permission from the journal.
} 
immediate effects of concussion on the adolescent brain and the nature of any long-lasting neuronal changes detectable with noninvasive multiparametric imaging.

In this study, concussed adolescent male hockey players were evaluated longitudinally after concussion and compared to independent, age-matched nonconcussed players as controls. While clinical measures in this population indicate recovery and prompt return to play, given previous imaging findings, we hypothesized that there would be persistent MRI-detectable neuronal changes that reflect long-lasting damage to white matter fibers and compensatory recovery mechanisms. Given this multiparametric MRI dataset, we were able to directly relate resting-state (RS-fMRI) changes with underlying structural abnormalities through diffusion tensor imaging (DTI), ${ }^{9}$ brain metabolic changes through magnetic resonance spectroscopy (MRS), and acute clinical deficits.

METHODS Players diagnosed with a mild traumatic brain injury or concussion (age $13.3 \pm 0.6$ years) were assessed within 24 to 72 hours of their injury $(n=17)$ and again 3 months later $(\mathrm{n}=14)$. An independent group of healthy, age-matched, nonconcussed male hockey players were assessed and acted as our controls ( $\mathrm{n}=26$, age $13.0 \pm 1$ years). Portions of this study design have been described previously. ${ }^{10}$ Further clinical methods are described in appendix e-1 at Neurology.org. The numbers of included participants for each portion of the study are summarized in table e-1. MRI acquisition parameters are outlined in appendix e-2.

Standard protocol approvals, registrations, and patient consents. Ethics approval was obtained through the University of Western Ontario's Health Sciences Research Ethics Board. All participants and parents/guardians provided informed written consent.

RS-fMRI analysis. RS-fMRI data were analyzed with FSL (FMRIB Software Library) 5.0.6 (Oxford, England) with 2 major aims: to investigate RS network (RSN) reorganization and wholebrain region-to-region connectivity patterns. Standard preprocessing steps included brain extraction, a high-pass filter (0.01 $\mathrm{Hz}$ ), spatial smoothing with a 5 -mm full-width half-maximum gaussian filter, and motion correction using linear image registration relative to the middle volume. Data with excessive motion (>1-mm maximum displacement or $>0.5-\mathrm{mm}$ relative mean displacement) were excluded from further analyses. Preprocessed images were denoised with single-session independent component analysis and automatic dimensionality estimation. Components that were designated as noise (related voxels outside the brain, within the corticospinal fluid, primarily high-frequency profile, or motion artifacts) were regressed from the data. Each dataset was registered to standard space with a 2 -stage affine registration technique that first registered the low-resolution functional data to the brain-extracted anatomic image and then to a 2-mm isotropic Montreal Neurologic Institute T1-weighted reference image using $12 d f$.
Multisession temporal concatenation-independent component analysis was used to create average RSN maps followed by dual regression to generate session-specific networks. ${ }^{11}$ In particular, the visual occipital pole, default mode (DMN), sensorimotor, executive control, and cerebellar networks were investigated. The FSL randomize permutation-testing tool was implemented to assess group differences according to a general linear model with threshold-free cluster enhancement and multiplecomparison Bonferroni-corrected $p$ values $(p<0.001)$. In addition, a regional connectivity analysis was performed on the preprocessed data with the CONN toolbox ${ }^{12}$ because of the statistical advantages as it complements the network analysis and expands to look at whole-brain regional connectivity. Participant-specific CSF and white matter masks were used as regressors. Data were parcellated into 136 functionally relevant regions with the Harvard-Oxford cortical atlas and cerebellar regions with the Automated Anatomical Labeling atlas. Wholebrain region-to-region connectivity differences were assessed with false discovery rate correction.

Diffusion tensor and tractography analysis. Raw diffusion datasets were corrected for eddy current distortions and relative head motion with the eddy command line tool. The nondiffusion-weighted $(b=0)$ volume was used to create a modestly extracted brain mask. A diffusion tensor was fit voxel-wise with the use of specific inputs of the gradient directions and $\mathrm{b}$ values $\left(b=1,000 \mathrm{~s} / \mathrm{mm}^{2}\right)$ directly from the scanner. Several diffusion metric spatial maps were created, including fractional anisotropy (FA) and mean (MD), axial (AD), and radial (RD) diffusivity. With the use of the John Hopkins University high-probability white matter tractography atlas, ${ }^{13}$ these DTI metrics were extracted and analyzed within the superior longitudinal fasciculus (SLF), cingulum, forceps major and minor of the corpus callosum, uncinate fasciculus, corticospinal tract (CST), anterior thalamic radiation, and inferior fronto-occipital and longitudinal fasciculus.

Once tracts with significant changes were identified with a multivariate analysis of variance, an iterative randomization tool within FSL was used to spatially quantify where the greatest changes occurred along those tracts while regressing for age. DTI metrics within the regions with the greatest changes were extracted and analyzed. These regions are referred to as $\mathrm{DTI}_{\max }$ and are described in Results. Probabilistic tractography with iterative Monte Carlo simulations of principal diffusion tensor vectors was used to quantify a probabilistic streamline between regions of interest (ROIs). Probabilistic structural connections were investigated between relevant parceled RS-fMRI regions to spatially relate structural and functional changes. To quantify these results, 2 identical spherical ROIs (5-mm radius) were placed before and after $\mathrm{DTI}_{\max }$ (that overlapped along the probabilistic tract connecting acute RS-fMRI ROIs that we identified, figure $1 \mathrm{~A}$ ). The probability distribution was displayed per participant and quantified the probability of a structural connection between the seed and waypoint masks (normalized by the total number of tracts modeled).

MRS analysis. Spectra were postprocessed with in-house software to measure absolute $\mathrm{N}$-acetylaspartate, choline, creatine, glutamate, glutamine, and myo-inositol as previously described. ${ }^{14-16}$ Briefly, analysis software created in our laboratory in IDL (version 5.4, Research Systems Inc, Boulder, CO) was used to correct spectral line shapes by combined QUALITY deconvolution and eddy current correction. ${ }^{17}$ Spectra were fitted in the time domain with the use of a Levenberg-Marquardt minimization routine ${ }^{14}$ with prior knowledge of metabolic line shapes. Prior knowledge was acquired from in vitro spectra obtained from aqueous solutions of metabolites before the study. ${ }^{14}$ We report absolute metabolite levels using unsuppressed 


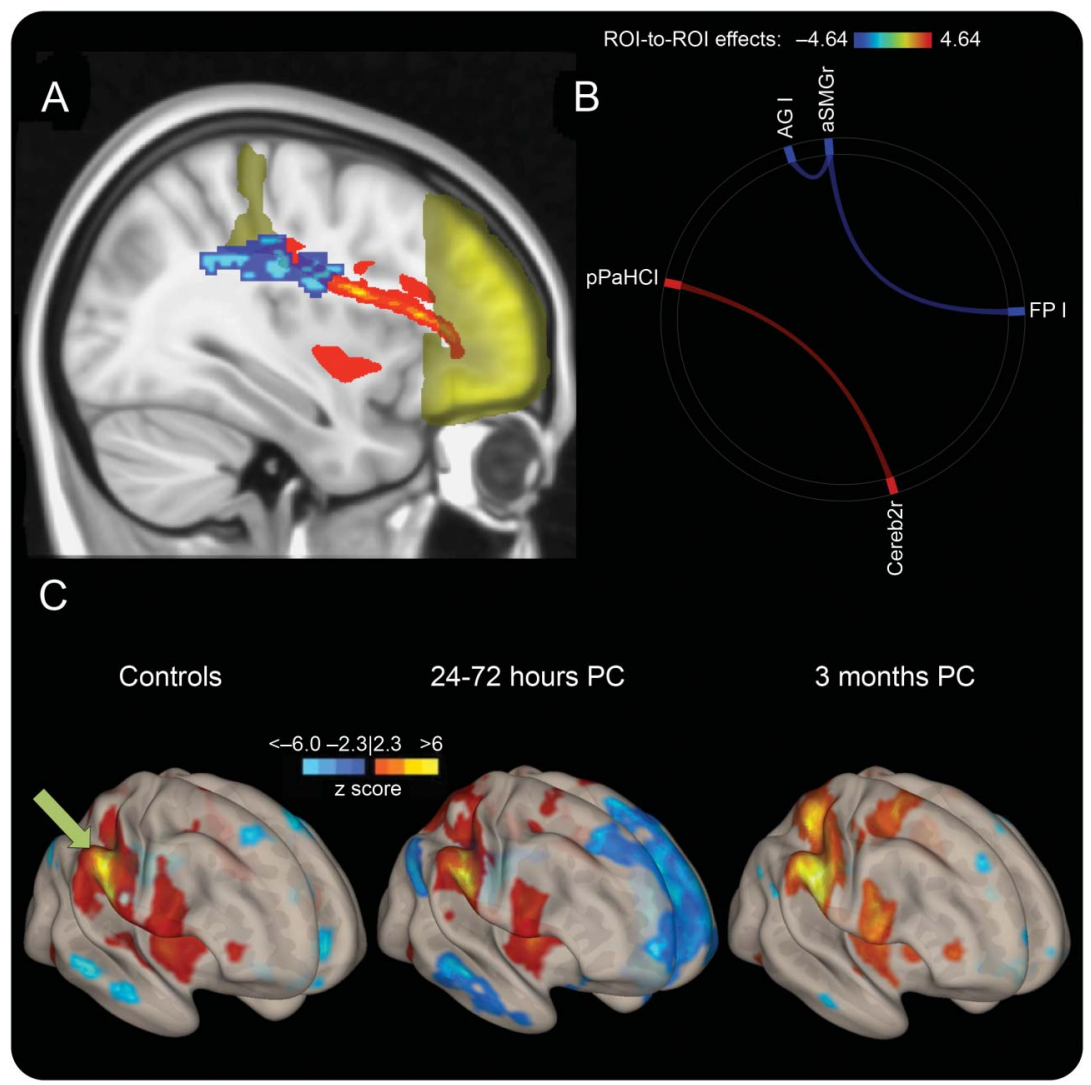

(A) Frontal pole (FP) and anterior supramarginal gyrus (aSMG) are shown in yellow, with the probabilistic tractography connecting them shown in red. DTI $\mathrm{max}_{\text {r }}$ region is overlaid in blue, showing the spatial relationship between structural abnormalities and resting-state fMRI connectivity. (B) Significant whole-brain region-to-region connectivity differences between controls and the 24- to 72-hour postconcussion (PC) group. Blue indicates a decrease and red indicates an increase in region-to-region connectivity. (C) Voxel-wise connectivity pattern using the right aSMG (green arrow) as a seed, displaying the enhanced anticorrelation (blue) with the FP and regions that mimic the default mode network. AG = angular gyrus; $\mathrm{pPaHC}=$ posterior parahippocampus; $\mathrm{ROI}=$ region of interest.

water from the ROI as an internal standard as previously described in detail. ${ }^{14,16}$ In addition, absolute concentrations included a correction for tissue partial volume and $\mathrm{T} 1$ and $\mathrm{T} 2$ relaxation-related signal loss.

Statistical analysis. Statistical analysis was performed with a multivariate analysis of variance with Tukey post hoc testing using GraphPad Prism (version 6.0). A hypothesis-driven 2tailed Pearson correlation analysis was explored between data that had a significant effect for group $(p<0.05$ after false discovery rate correction).

RESULTS Clinical results are summarized in appendix e-3 and figure e-1.

RS-fMRI results. We found significant increases in connectivity at 3 months after concussion in several RSNs. In particular, there was significantly increased connectivity at 3 months after concussion compared to controls with the occipital pole visual network and cerebellar network (figure 2, B and C). Compared to 24 to 72 hours after concussion, there were significant increases in connectivity by 3 months with the sensorimotor network (figure 2D) and cerebellar network.
There was increased DMN connectivity at 3 months compared to controls that did not survive statistical Bonferroni correction ( $p<0.01$, figure $2 \mathrm{~A}$ ), and no significant differences were found within the executive control network or between controls and the 24- to 72-hour postconcussion group in any network.

There were a few subtle changes in region-toregion connectivity within 24 to 72 hours after concussion compared to controls (figure 1B); however, some were striking with a large effect size $(>0.8)$ and prompted further analysis to directly relate with our DTI findings (figure 1C). Specifically, there were significant anticorrelations between the anterior supramarginal gyrus and areas that mimic the DMN, including the angular gyrus and frontal pole $(p<$ 0.05 , including portions of the medial prefrontal cortex, figure 1C). This was not found in our network analysis, possibly as a result of statistical power (voxellevel correction) or the dominance of the frontal pole. There was an increase in regional connectivity immediately after concussion compared to controls 


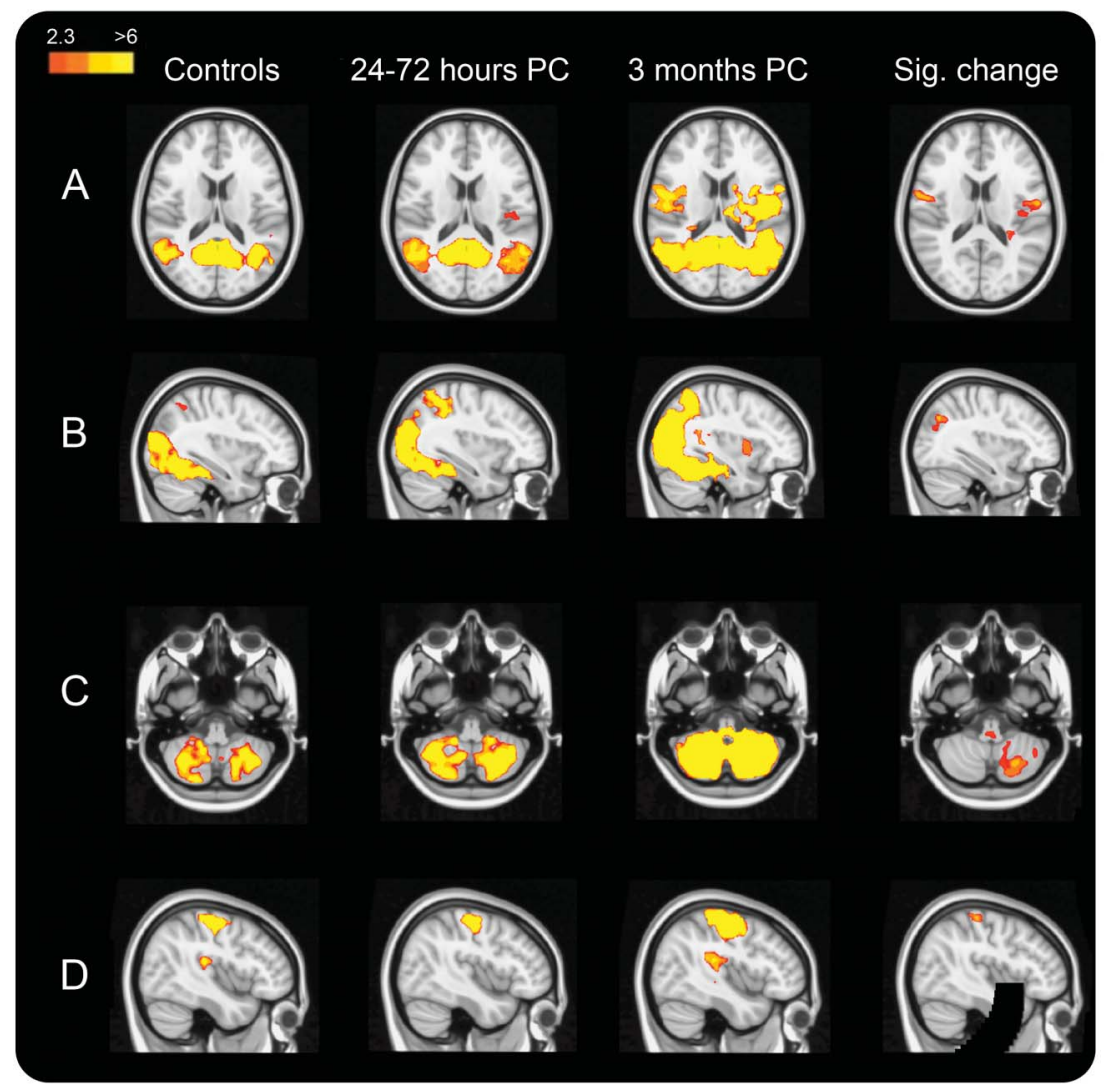

Each column represents the average resting-state network (RSN) for each of the 3 groups (scaled by $z$ statistic), with areas that are significantly more highly correlated with that network at 3 months compared to (A-C) controls or (D) the 24- to 72hour postconcussion (PC) group ( $p<0.001$ except for $[A]$ where $p<0.01$ ). Results are shown for the (A) default mode network, (B) occipital pole visual RSN, (C) cerebellar RSN, and (D) sensorimotor RSN.

between the cerebellum and parahippocampal gyrus. The significant whole-brain region-to-region connectivity changes are shown in figure 3 , and similar to the RSN results, there were increases in connectivity at 3 months compared to both the control and 24- to 72hour postconcussion groups between regions that complimented our network-level connectivity changes.

Diffusion results. There was a significant main effect for group differences in the CST, cingulum, and SLF $(F>4.18, p<0.05$, figure 4). Along the CST, the greatest diffusion changes were located inferiorly near the brainstem $(p<0.1)$, and there were small, more scattered maximums along the cingulum $(p>0.1)$. A large region along the right SLF shown in figure 1 had significant $\mathrm{MD}, \mathrm{RD}$, and $\mathrm{AD}$ changes at 3 months after concussion $(p<0.01)$, and a mask was created over this localized hotspot of compromised neuronal integrity, DTI $\mathrm{max}_{\max }$. Within $\mathrm{DTI}_{\max }$, the $\mathrm{MD}$ and $\mathrm{RD}$ values were significantly decreased at both 24 to 72 hours and 3 months after concussion compared to controls $(F>10.0, p<0.01)$; furthermore, there were decreases in $\mathrm{AD}$ at both time points
( $F=6.67, p=0.060$ and $p=0.003$, respectively) with increases in FA by 3 months $(F=3.91, p=$ $0.025)$. Using the anterior supramarginal gyrus as a seed and the frontal pole as a waypoint, we confirmed that probabilistic tracts structurally connected regions where we found acute RS-fMRI differences (figure 1). The probability of a structural connection did not significantly decrease after concussion.

Spectroscopy results. The spectroscopy voxel was placed in the prefrontal region with the following mean \pm SD tissue content: gray matter 19\% \pm $7 \%$, white matter $78 \% \pm 8 \%$, and CSF $3 \% \pm$ $2 \%$. The tissue composition of the voxel did not significantly change across participants or sessions.

No changes in $N$-acetylaspartate were found between any of the groups. Choline was significantly reduced at 3 months relative to controls $(F=4.2$, $p=0.02$, figure 4$)$, with a $10 \%$ decrease in the mean observed $(p=0.035)$. A reduction in glutamine levels was observed but was not significantly different from controls $(F=2.3, p=0.09)$. Correlation results are summarized in detail in table e-2, and examples are shown in figure 5 . 


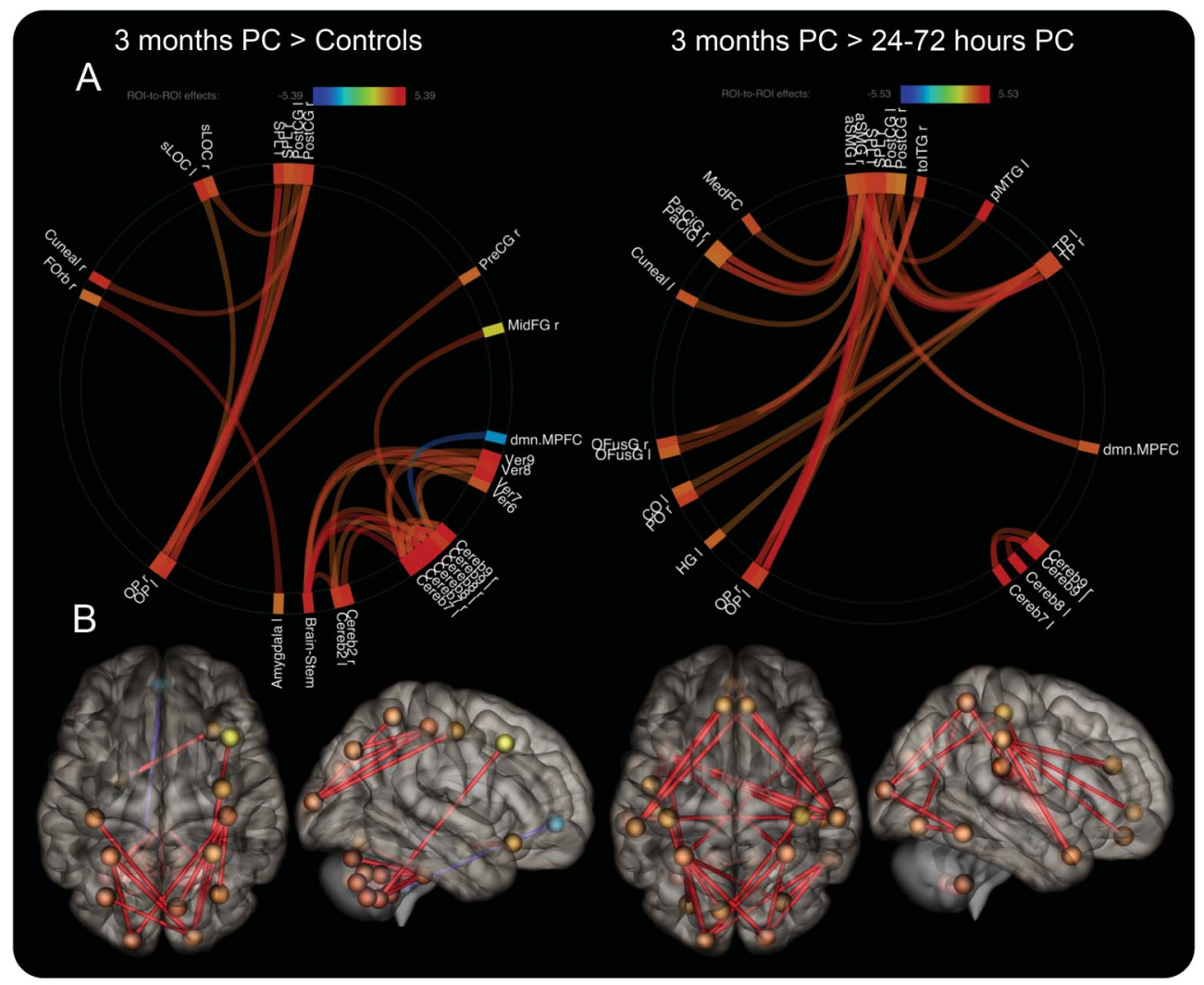

Significantly increased connectivity (red lines) is shown using (A) connectome rings with regions labeled around the perimeter and (B) 3-dimensional brain volumes in which the color of the spheres and the transparency of the connecting lines indicate the strength of the effect using a t statistic after false discovery rate correction (corrected $p<0.05$ ). aSMG $=$ anterior supramarginal gyrus; Cereb $=$ cerebellum; $\mathrm{CO}=$ central opercular cortex; dmn.MPFC $=$ default mode network medial prefrontal cortex; HG = Heschl gyrus; MedFC = medial frontal cortex; MidFG = middle frontal gyrus; OFusG = occipital fusiform gyrus; $\mathrm{OP}=$ occipital pole; $\mathrm{PaCiG}=$ paracingulate gyrus; $\mathrm{pMTG}=$ posterior middle temporal gyrus; $\mathrm{PO}=$ parietal operculum cortex; Pre/PostCG = precentral/postcentral gyri; sLOC = superior lateral occipital cortex; SPL = superior parietal lobule; tolTG = temporoccipital inferior temporal gyrus; TP = temporal pole; Ver = vermis.

DISCUSSION In this study of adolescent hockey players from Bantam leagues in which body checking is first introduced, we found acute and longitudinal multiparametric MRI changes after concussion compared to controls. As expected, clinical composite scores elevated immediately after concussion, and within weeks of injury, they returned to control levels and players were cleared to return to play. However, we found persistent RS-fMRI connectivity changes, diffusion-related white matter abnormalities, and MRS metabolite decreases in the prefrontal white matter at 3 months after concussion.

The adolescent brain is still not fully developed during this age range (11-14 years), and as it continues to grow and mature, it may be more vulnerable to brain dysfunction and elongated periods of recovery after an acceleration-related injury. It has been shown that this heightened vulnerability may be due to both biomechanical (neck strength) and neurobiological (incomplete white matter myelination) characteristics. ${ }^{18}$ Axonal excitability and electrophysiologic recordings in a rat fluid percussion brain injury model have been used to assess differences in axonal vulnerability between myelinated and unmyelinated fibers during injury response and functional recovery. ${ }^{19}$ In this animal model, myelinated axons recovered within 7 days and were relatively protected compared to unmyelinated fibers, which were more severely injured and took longer to recover. It is critical to understand how the adolescent human brain reacts and recovers from concussion. A recent review of concussion-related MRI findings demonstrated the advantages of relating multiple MRI modalities, ${ }^{20}$ and here we aim to do just that to better understand the meaning of these changes.

We found diffuse changes in DTI metrics along 3 major white matter tracts. However, the tract-specific spatial changes were localized to a single, central region along the SLF $\left(\mathrm{DTI}_{\max }\right)$ where we found decreased $\mathrm{AD}$, $\mathrm{MD}$, and $\mathrm{RD}$ at both times after concussion. In vivo animal studies have been able to directly relate histology with MRI diffusion, specifically finding a direct 


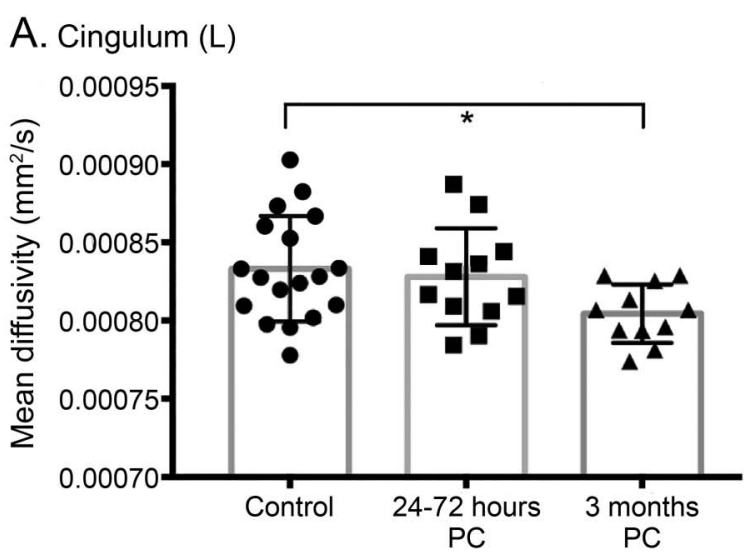

C. Corticospinal tract (R)

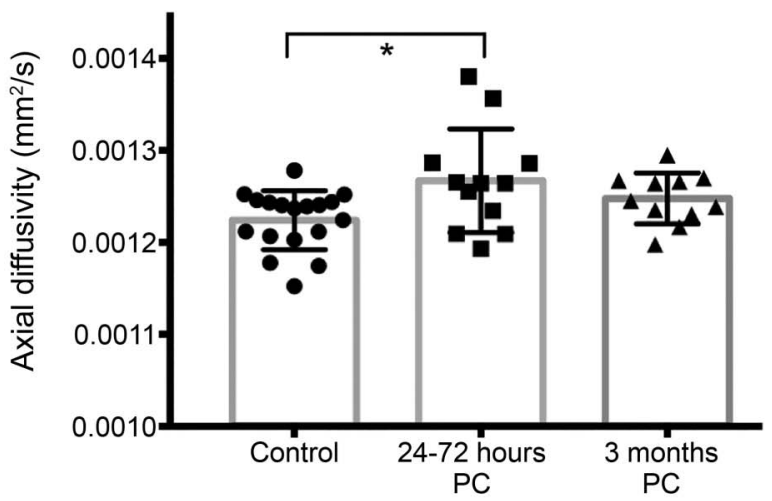

E. DTI $I_{\text {max }}$ region of interest

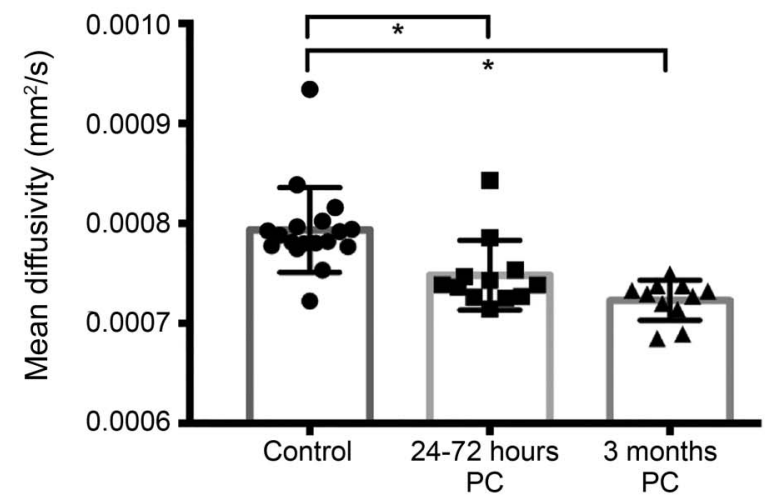

B. Superior longitudinal fasciculus $(R)$

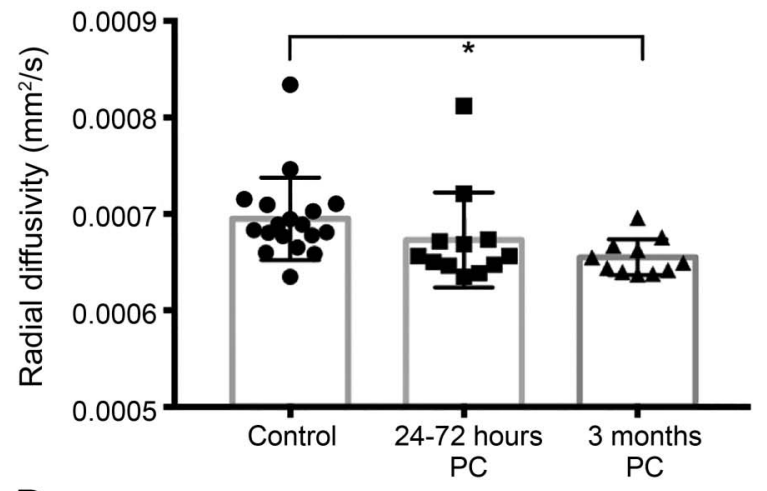

D. DTI ${ }_{\max }$ region of interest

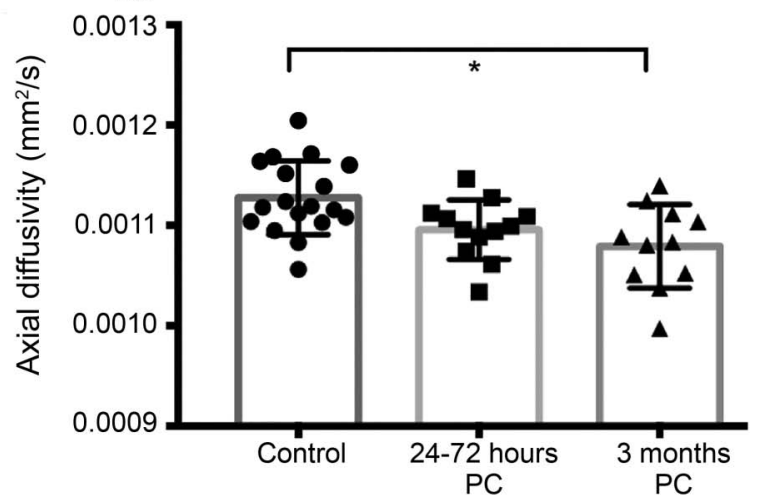

F. Choline (CHO)

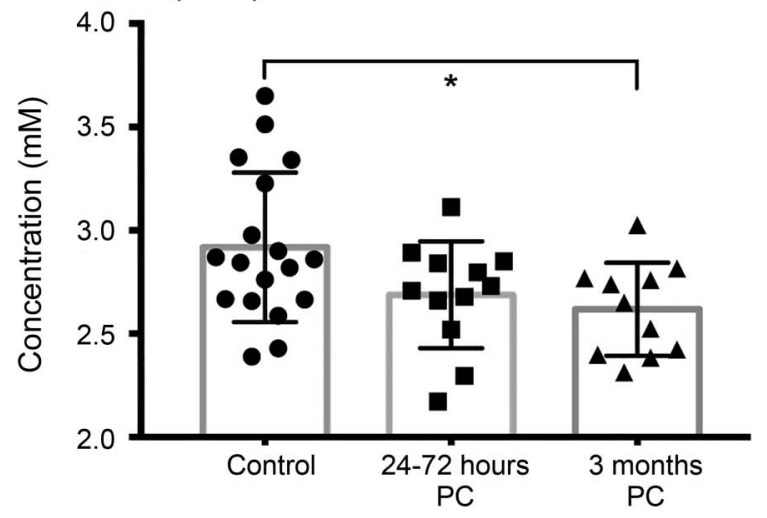

(A-E) Average diffusion metrics within standard atlas-derived white matter tracts and (F) absolute metabolite concentrations with the prefrontal white matter voxel. SDs are shown with error bars. *Significant multivariate analysis of variance-corrected post hoc differences $(p<0.05)$. DTI $=$ diffusion tensor imaging; MRS = magnetic resonance spectroscopy; $\mathrm{PC}=$ postconcussion.

relationship between $\mathrm{AD}$ and axonal injury through postmortem neurofilament immunostaining and confirmation of the presence of $\beta$-amyloid precursor protein. ${ }^{21}$ Diffuse axonal pathology has been observed in all traumatic brain injury severities, in which transport is disrupted and axonal bulb formations occur. ${ }^{22}$ The decreases in $\mathrm{AD}$ observed after concussion may reflect this axonal pathology because they relate directly to immediate symptoms. The $\mathrm{AD}$ within $\mathrm{DTI}_{\max }$ at 24 to 72 hours after concussion was significantly correlated with total symptom scores and the number of errors during balance testing, indicating that a more severe injury is associated with more severe axonal damage. This remained true at 3 months after concussion when the sustained decrease in $\mathrm{AD}$ within $\mathrm{DTI}_{\max }$ was associated with a higher symptom severity score.

The most common injury mechanism in our cohort was falling, affecting the back of the head. Investigations of the biomechanical deformations and shearing forces after even minimal acceleration of the human head have reported deformation of the human brain. Rapid deceleration directed to the back 
A. Correlation at $24-72$ hours PC

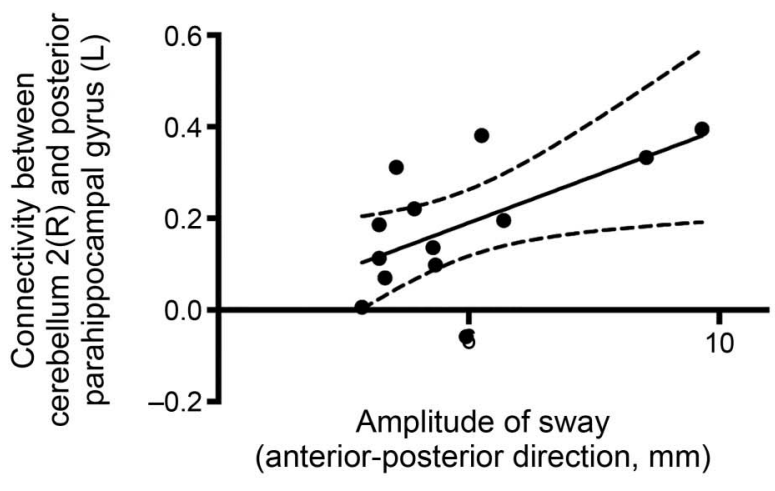

C. Correlation at 3 months $\mathrm{PC}$
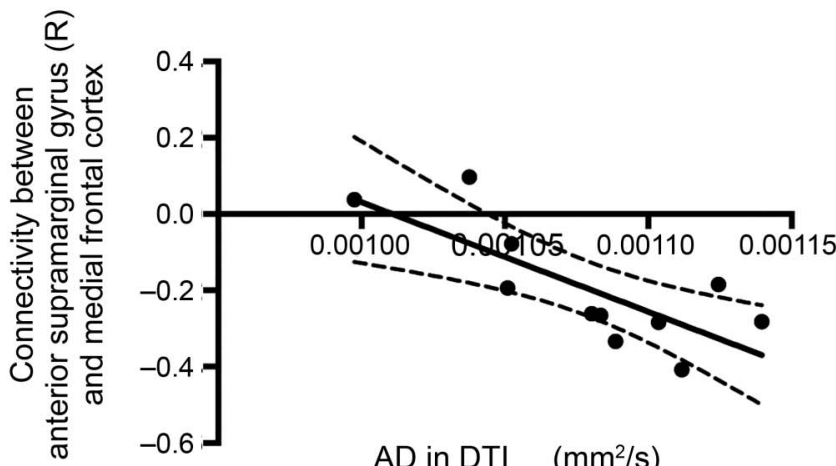

$\mathrm{AD}$ in $\mathrm{DTI}_{\max }\left(\mathrm{mm}^{2} / \mathrm{s}\right)$
B. Correlation at 24-72 hours PC

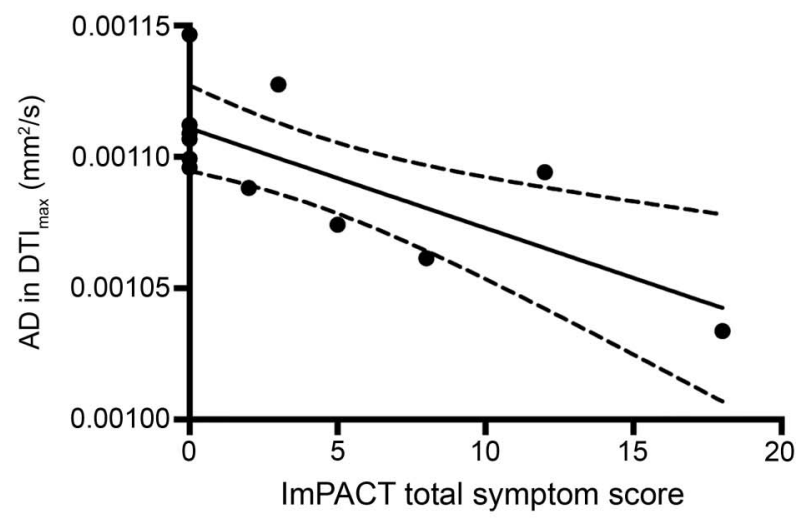

D. Correlation at 3 months PC

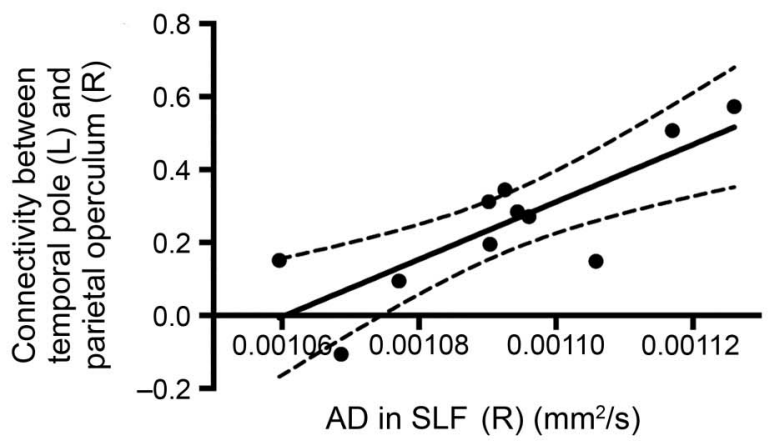

E. Correlatng data $24-72$ hours PC (x-axis) with 3 month PC data (y-axis)
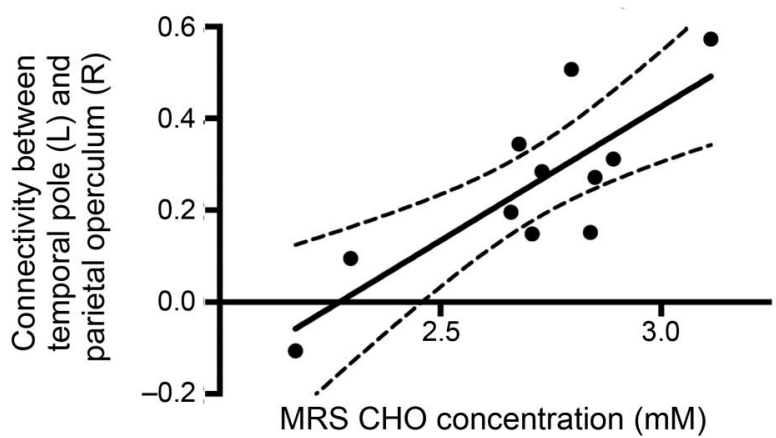

\section{F. Correlating data $24-72$ hours PC (x-axis) with 3 month PC data (y-axis)}
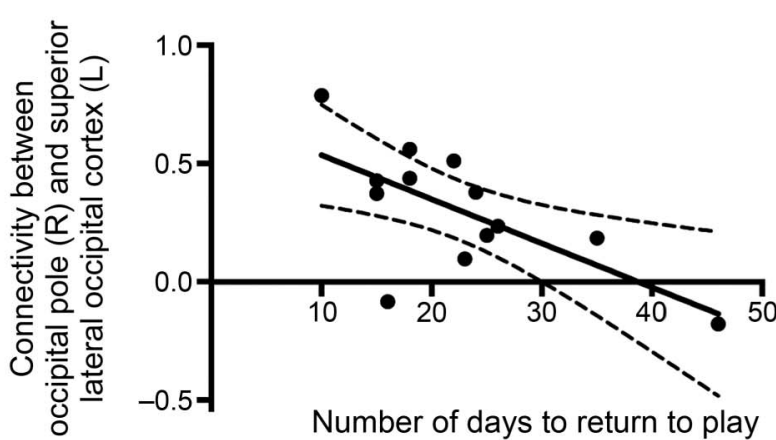

Relating data at ( $A$ and $B) 24$ to 72 hours postconcussion (PC), (C and D) 3 months PC, and ( $E$ and $F$ ) between 24 to 72 hours $P C$ ( $x$-axis) and 3 months $P C$ ( $y$ axis) data. Further details are provided in table e-2. The $95 \%$ confidence interval is shown using the dashed curves, and all relationships shown here have $p<$ 0.05 after false discovery rate correction. $\mathrm{AD}=$ axial diffusivity; $\mathrm{CHO}=$ choline; $\mathrm{DTI}=$ diffusion tensor imaging; ImPACT = Immediate Post-Concussion Assessment and Cognitive Testing; MRS = magnetic resonance spectroscopy; SLF = superior longitudinal fasciculus.

of the head results in anterior shortening and posterior elongation, as well as deformations in the axial plane. ${ }^{23}$ These results correspond to regions where we found diffusion changes indicative of structural damage to the long tracts running anterior to posterior. This acceleration injury and the consequent strain and shearing forces between brain tissues may damage axons themselves. The decrease in $\mathrm{AD}$ was concurrent with decreases in $\mathrm{RD}$ and $\mathrm{MD}$ and increases in FA within the same region. This could reflect different pathologies such as axonal disruption, cytotoxic edema, axonal swelling, or changes in myelin at the site of injury. ${ }^{24}$ These findings are similar to some previous DTI studies ${ }^{5,9,25-27}$ but not all. ${ }^{28,29}$ Cohorts of different ages, injury severity, and acquisition timing all may play a role in the direction of these changing 
diffusion metrics. Fluid-attenuated inversion recovery and turbo spin echo sequences were used to confirm a mild traumatic brain injury with no cerebral edema. We observed region-to-region RS-fMRI hyperconnectivity at 3 months after concussion compared to both controls and 24 to 72 hours after concussion. This was also true at the network level. Previous studies have variably found this pattern, and hyperconnectivity has been proposed to be involved in recovery and compensation for underlying white matter disruption. ${ }^{4,30-33}$

Here, we demonstrate the strength of multiparametric MRI by relating our connectivity findings directly to DTI and clinical data. Acute regional connectivity changes with the cerebellum correlated with all of the significant measures reflecting acute postural balance deficits and directly relate the role of the cerebellum in balance performance. ${ }^{34}$ The average AD along the SLF and within DTI max $_{\text {was }}$ warrelated with changes in interregional connectivity in which, in general, more axonal injury (lower diffusivity) and a more severe injury as quantified by number and severity of symptoms or more days required to recover were associated with lower absolute values of connectivity. Only those players with a less severe injury as indicated by these acute clinical measures exhibited regional hyperconnectivity at 3 months after concussion, similar to previous work focused on the DMN in more severe and older patients with traumatic brain injury 6 months after their injury. ${ }^{35}$ This possible recovery mechanism may be compensating for the underlying damaged avenues of structural connections by recruiting regions of connectivity and enhancing both correlated and inhibitory communication. It is possible that some players have not yet reached or completed the recovery phase, and further longitudinal data are needed to understand the timing and potential long-term consequences of these mechanisms.

A $10 \%$ reduction in the MRS choline signal was observed at 3 months relative to controls. The choline signal is made up of different components, mainly phosphorylcholine (a precursor to membrane synthesis) and glycerophosphorylcholine (a breakdown product of the membrane). ${ }^{36}$ This change is particularly interesting because reductions in blood plasma glycerophospholipids at 24 to 72 hours were reported in a subset of these hockey players. ${ }^{10}$ Other studies have also reported reduced choline levels in the motor cortex after a month of high school football in nonconcussed players. ${ }^{37}$ This was suggested to be due to a decrease in membrane turnover as a result of repetitive subconcussive impacts. The correlations with the MRS data support similar interpretations. The choline concentration at both 24 to 72 hours and 3 months after concussion was significantly correlated with RS-fMRI connectivity; in particular, a lower choline concentration was associated with a lower absolute interregional connectivity value. Given the relationship between choline concentrations and the clinical, diffusion, and connectivity measures, we hypothesize that the decrease in choline is related to reduced membrane turnover rate. ${ }^{36}$

There are limitations in the present study that will motivate future MRI investigations of adolescent concussion. The average changes we report here represent a heterogeneous population whose individual injury mechanisms, severities, and recovery times vary. We followed up with players 3 months after their injury; however, the players had clinically recovered and were cleared to return to play at different times. It is possible that returning to play may affect the brain and any ongoing neuroreparative mechanisms. Given the long-lasting changes we found at 3 months, future longitudinal studies should continue to follow this demographic as they develop to understand exactly how long the recovery period lasts as a function of age and the consequences, if any, later in life, including increased vulnerability to future concussions and the development of neurodegenerative diseases such as chronic traumatic encephalopathy. ${ }^{38,39}$

The persistent changes we observed in this study provide evidence of prolonged axonal disruption in a localized area along the SLF. This result was spatially related to acute RS-fMRI connectivity changes and choline metabolite decreases and correlated significantly with clinical deficits. Diffuse hyperconnectivity patterns were present 3 months after concussion, well after clearance to return to play and symptomatic recovery. This adolescent population may be particularly vulnerable to injury while axons continue to myelinate and mature, and our results suggest that they may require longer recovery periods.

\section{AUTHOR CONTRIBUTIONS}

All authors approved the manuscript and provided critical edits. K.Y.M: manuscript preparation, analysis and interpretation of data, statistical analysis. A.S.: analysis and interpretation of data. R.B.: study concept and design. G.A.D: overall project design. C.B.: study coordinator. A.B.: overall project design. L.F.: overall project design, patient evaluation. K.A.: patient evaluation. T.J.D.: interpretation of data. D.D.F.: overall project design. J.H.: overall project design, clinical acquisition. R.S.M.: overall project and image acquisition design, interpretation of data.

\section{ACKNOWLEDGMENT}

The authors acknowledge Sandra Shaw for her efforts in organizing patient appointments. They also thank and commend the players and their parents/guardians for their willingness to participate in this study and the coaches and MRI technicians for their excellent support.

\section{STUDY FUNDING}

Funding was provided by a grant from the Children's Health Foundation to Dr. D.D. Fraser and a grant from the Western University Schulich School of Medicine and Dentistry (co-principal investigators: Dr. D.D. Fraser and G.A. Dekaban). 


\section{DISCLOSURE}

K. Manning and A. Schranz report no disclosures relevant to the manuscript. R. Bartha is funded by CIHR Project Grant 152927, Canadian Consortium on Neurodegeneration in Aging 003658, Natural Sciences and Engineering Research Council of Canada grant 250313-2013, Ontario Institute for Cancer Research Grant 00807 Smarter Imaging Program, Ontario Brain Institute grant ONDRI2013, and an Alzheimer Foundation of London and Middlesex Premier Research Grant. G. Dekaban is funded by CIHR project grant PJT 148651, is co-principal investigator on the London Health Sciences Centre Children's Health Foundation grant LHR F7806 R-13-116 CHRI (funded the work presented in this publication), is co-principal investigator or applicant on 3 additional CIHR grants, and receives funds from the NSE BioCanRx. C. Barreira, A. Brown, L. Fischer, and K. Asem report no disclosures relevant to the manuscript. T. Doherty receives research support from Allergan Canada. D. Fraser receives funding through the Children's Health Foundation. J. Holmes is funded by CIHR operating grant 326065 and an Alzheimer's Association International Research Grant. R. Menon is funded by CIHR Foundation Grant 353372, Natural Sciences and Engineering Research Council of Canada grant 261701-2012, and a Brain Canada Platform Support Grant; has sold a patent to Siemens Healthcare; and receives research support from Siemens Healthineers. Go to Neurology.org for full disclosures.

Received April 19, 2017. Accepted in final form September 6, 2017.

\section{REFERENCES}

1. Benz B, Ritz A, Kiesow S. Influence of age-related factors on long-term outcome after traumatic brain injury (TBI) in children: a review of recent literature and some preliminary findings. Restor Neurol Neurosci 1999;14:135-141.

2. Cusimano MD, Cho N, Amin K, et al. Mechanisms of team-sport-related brain injuries in children 5 to 19 years old: opportunities for prevention. PLoS One 2013;8: e58868.

3. Bigler ED, Maxwell WL. Neuropathology of mild traumatic brain injury: relationship to neuroimaging findings. Brain Imaging Behav 2012;6:108-136.

4. Borich M, Babul AN, Yuan PH, Boyd L, Virji-Babul N. Alterations in resting-state brain networks in concussed adolescent athletes. J Neurotrauma 2015;32:265-271.

5. Mayer AR, Ling JM, Yang Z, Pena A, Yeo RA, Klimaj S. Diffusion abnormalities in pediatric mild traumatic brain injury. J Neurosci 2012;32:17961-17969.

6. Maugans TA, Farley C, Altaye M, Leach J, Cecil KM. Pediatric sports-related concussion produces cerebral blood flow alterations. Pediatrics 2012;129:28-37.

7. Westfall DR, West JD, Bailey JN, et al. Increased brain activation during working memory processing after pediatric mild traumatic brain injury (mTBI). J Pediatr Rehabil Med 2015;8:297-308.

8. Kirkwood MW, Yeates KO, Wilson PE. Pediatric sportrelated concussion: a review of the clinical management of an oft-neglected population. Pediatrics 2006;117:13591371.

9. Bazarian JJ, Zhong J, Blyth B, Zhu T, Kavcic V, Peterson D. Diffusion tensor imaging detects clinically important axonal damage after mild traumatic brain injury: a pilot study. J Neurotrauma 2007;24:1447-1459.

10. Daley M, Dekaban G, Bartha R, et al. Metabolomics profiling of concussion in adolescent male hockey players: a novel diagnostic method. Metabolomics 2016;12:185.

11. Filippini N, MacIntosh BJ, Hough MG, et al. Distinct patterns of brain activity in young carriers of the APOEepsilon4 allele. Proc Natl Acad Sci USA 2009;106:72097214.
12. Whitfield-Gabrieli S, Nieto-Castanon A. Conn: a functional connectivity toolbox for correlated and anticorrelated brain networks. Brain Connect 2012;2:125-141.

13. Mori S, Oishi K, Faria AV. White matter atlases based on diffusion tensor imaging. Curr Opin Neurol 2009;22: 362-369.

14. Bartha R, Drost DJ, Williamson PC. Factors affecting the quantification of short echo in-vivo $1 \mathrm{H}$ MR spectra: prior knowledge, peak elimination, and filtering. NMR Biomed 1999;12:205-216.

15. Kowalczyk I, Duggal N, Bartha R. Proton magnetic resonance spectroscopy of the motor cortex in cervical myelopathy. Brain 2012;135:461-468.

16. Goncalves S, Stevens TK, Doyle-Pettypiece P, Bartha R, Duggal N. N-acetylaspartate in the motor and sensory cortices following functional recovery after surgery for cervical spondylotic myelopathy. J Neurosurg Spine 2016;25: 436-443.

17. Bartha R, Drost DJ, Menon RS, Williamson PC. Spectroscopic lineshape correction by QUECC: combined QUALITY deconvolution and eddy current correction. Magn Reson Med 2000;44:641-645.

18. Toledo E, Lebel A, Becerra L, et al. The young brain and concussion: imaging as a biomarker for diagnosis and prognosis. Neurosci Biobehav Rev 2012;36: 1510-1531.

19. Reeves TM, Phillips LL, Povlishock JT. Myelinated and unmyelinated axons of the corpus callosum differ in vulnerability and functional recovery following traumatic brain injury. Exp Neurol 2005;196:126-137.

20. Shin SS, Bales JW, Edward Dixon C, Hwang M. Structural imaging of mild traumatic brain injury may not be enough: overview of functional and metabolic imaging of mild traumatic brain injury. Brain Imaging Behav 2017; 11:591-610.

21. MacDonald CL, Dikranian K, Song SK, Bayly PV, Holtzman DM, Brody DL. Detection of traumatic axonal injury with diffusion tensor imaging in a mouse model of traumatic brain injury. Exp Neurol 2007;205:116-131.

22. Johnson VE, Stewart W, Smith DH. Axonal pathology in traumatic brain injury. Exp Neurol 2014;246:35-43.

23. Bayly PV, Cohen TS, Leister EP, Ajo D, Leuthardt EC, Genin GM. Deformation of the human brain induced by mild acceleration. J Neurotrauma 2005;22:845-856.

24. Wright $\mathrm{AD}$, Jarrett $\mathrm{M}$, Vavasour I, et al. Myelin water fraction is transiently reduced after a single mild traumatic brain injury: a prospective cohort study in collegiate hockey players. PLoS One 2016;11:e0150215.

25. Chu Z, Wilde EA, Hunter JV, et al. Voxel-based analysis of diffusion tensor imaging in mild traumatic brain injury in adolescents. Am J Neuroradiol 2010;31: 340-346.

26. Lancaster MA, Olson DV, McCrea MA, Nelson LD, LaRoche AA, Muftuler LT. Acute white matter changes following sport-related concussion: a serial diffusion tensor and diffusion kurtosis tensor imaging study. Hum Brain Mapp 2016;37:3821-3834.

27. Wilde EA, McCauley SR, Hunter JV, et al. Diffusion tensor imaging of acute mild traumatic brain injury in adolescents. Neurology 2008;70:948-955.

28. Koerte IK, Kaufmann D, Hartl E, et al. A prospective study of physician-observed concussion during a varsity university hockey season: white matter integrity in ice hockey players, part 3 of 4. Neurosurg Focus 2012;33:E3. 
29. Goetz P, Blamire A, Rajagopalan B, Cadoux-Hudson T, Young D, Styles P. Increase in apparent diffusion coefficient in normal appearing white matter following human traumatic brain injury correlates with injury severity. J Neurotrauma 2004;21:645-654.

30. Czerniak SM, Sikoglu EM, Liso Navarro AA, et al. A resting state functional magnetic resonance imaging study of concussion in collegiate athletes. Brain Imaging Behav 2014;9:323-332.

31. Hillary FG, Slocomb J, Hills EC, et al. Changes in resting connectivity during recovery from severe traumatic brain injury. Int J Psychophysiol 2011;82:115-123.

32. Iraji A, Benson RR, Welch RD, et al. Resting state functional connectivity in mild traumatic brain injury at the acute stage: independent component and seed based analyses. J Neurotrauma 2014;1045:1-57.

33. Tang CY, Eaves E, Dams-O'Connor K, et al. Diffuse disconnectivity in $\mathrm{tBi}$ : a resting state $\mathrm{fMri}$ anD Dti study. Transl Neurosci 2012;3:9-14.
34. Morton SM, Bastian AJ. Cerebellar control of balance and locomotion. Neurosci 2004;10:247-259.

35. Sharp DJ, Beckmann CF, Greenwood R, et al. Default mode network functional and structural connectivity after traumatic brain injury. Brain 2011;134: 2233-2247.

36. Maddock RJ, Buonocore MH. MR spectroscopic studies of the brain in psychiatric disorders. Curr Top Behav Neurosci 2012;11:199-251.

37. Poole VN, Breedlove EL, Shenk TE, et al. Sub-concussive hit characteristics predict deviant brain metabolism in football athletes. Dev Neuropsychol 2015;40:12-17.

38. Anderson V, Catroppa C, Morse S, Haritou F, Rosenfeld J. Functional plasticity or vulnerability after early brain injury? Pediatrics 2005;116:1374-1382.

39. Daneshvar DH, Riley DO, Nowinski CJ, McKee AC, Stern RA, Cantu RC. Long-term consequences: effects on normal development profile after concussion. Phys Med Rehabil Clin N Am 2011;22:683-700.

\section{This Week's Neurology ${ }^{\circledR}$ Podcast}

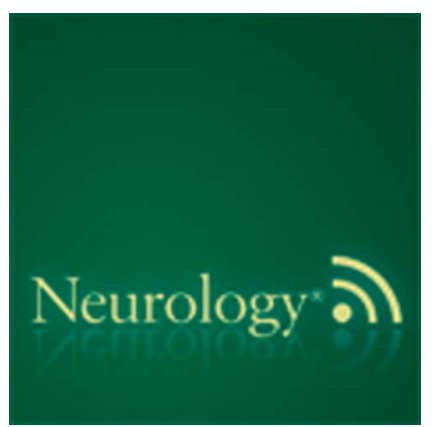

Multiparametric MRI changes persist beyond recovery in concussed adolescent hockey players (see p. 2157)

This podcast begins and closes with Dr. Robert Gross, Editor-inChief, briefly discussing highlighted articles from the November 21, 2017, issue of Neurology. In the first segment, Dr. Ted Burns talks with Dr. Kathryn Manning and Dr. Ravi Menon about their paper regarding the effects of concussion on adolescent hockey players. In the second part of the podcast, Dr. Burns focuses his interview with Dr. Christopher Goodman on a recent Neurology Today article featuring Dr. Goodman, which discussed the increase in gabapentinoid prescription.

Disclosures can be found at Neurology.org.

At Neurology.org, click on "RSS" in the Neurology Podcast box to listen to the most recent podcast and subscribe to the RSS feed.

CME Opportunity: Listen to this week's Neurology Podcast and earn 0.5 AMA PRA Category 1 CME Credits ${ }^{\mathrm{TM}}$ by answering the multiple-choice questions in the online Podcast quiz.

\section{Get Connected. Stay Connected.}

Connect with the American Academy of Neurology's popular social media channels to stay up-todate on the latest news and breakthroughs in neurology, and network with peers and neurology thought leaders. Visit $A A N$.com/Connect. 


\section{Neurology}

Multiparametric MRI changes persist beyond recovery in concussed adolescent hockey players

Kathryn Y. Manning, Amy Schranz, Robert Bartha, et al.

Neurology 2017;89;2157-2166 Published Online before print October 25, 2017

DOI 10.1212/WNL.0000000000004669

This information is current as of October 25, 2017

Neurology ${ }^{\circledR}$ is the official journal of the American Academy of Neurology. Published continuously since 1951, it is now a weekly with 48 issues per year. Copyright Copyright $@ 2017$ The Author(s). Published by Wolters Kluwer Health, Inc. on behalf of the American Academy of Neurology.. All rights reserved. Print ISSN: 0028-3878. Online ISSN: 1526-632X.

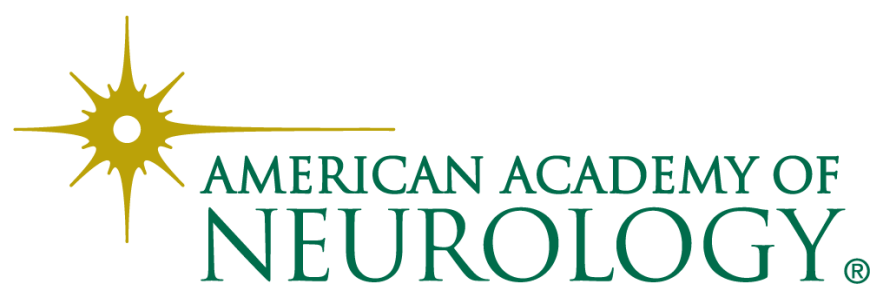




\section{Updated Information \& Services}

\section{Supplementary Material}

\section{References}

Citations

Subspecialty Collections

Errata

Permissions \& Licensing

Reprints including high resolution figures, can be found at: http://n.neurology.org/content/89/21/2157.full

Supplementary material can be found at: http://n.neurology.org/content/suppl/2017/10/25/WNL.0000000000004 669.DC1

http://n.neurology.org/content/suppl/2017/11/27/WNL.0000000000004 669.DC2

This article cites 39 articles, 4 of which you can access for free at: http://n.neurology.org/content/89/21/2157.full\#ref-list-1

This article has been cited by 3 HighWire-hosted articles: http://n.neurology.org/content/89/21/2157.full\#\#otherarticles

This article, along with others on similar topics, appears in the following collection(s):

\section{Brain trauma}

http://n.neurology.org/cgi/collection/brain_trauma

\section{DWI}

http://n.neurology.org/cgi/collection/dwi

\section{fMRI}

http://n.neurology.org/cgi/collection/fmri

MRI

http://n.neurology.org/cgi/collection/mri

MRS

http://n.neurology.org/cgi/collection/mrs

An erratum has been published regarding this article. Please see next page or:

/content/90/22/1039.full.pdf

Information about reproducing this article in parts (figures,tables) or in its entirety can be found online at:

http://www.neurology.org/about/about_the_journal\#permissions

Information about ordering reprints can be found online:

http://n.neurology.org/subscribers/advertise

Neurology ${ }^{\circledR}$ is the official journal of the American Academy of Neurology. Published continuously since 1951, it is now a weekly with 48 issues per year. Copyright Copyright ( 2017 The Author(s). Published by Wolters Kluwer Health, Inc. on behalf of the American Academy of Neurology.. All rights reserved. Print ISSN: 0028-3878. Online ISSN: 1526-632X.

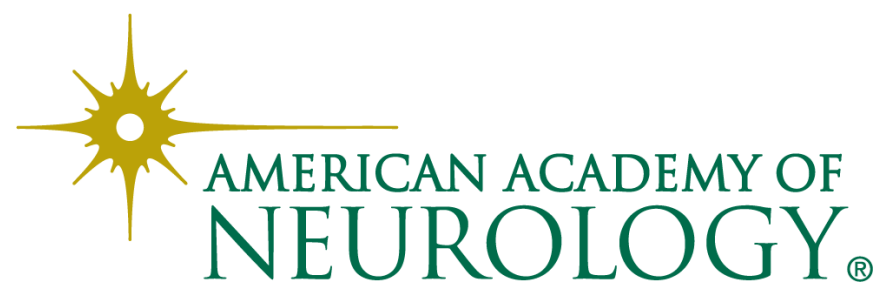




\section{Disputes \& Debates: Editors' Choice}

Steven Galetta, MD, FAAN, Section Editor

\section{Editors' note: Pilocytic astrocytoma with anaplasia arising from the optic chiasm in a very elderly patient}

In reference to the NeuroImage "Pilocytic astrocytoma with anaplasia arising from the optic chiasm in a very elderly patient," Dr. Wasilewski comments that there are few studies and no standard of care guidelines in the treatment of pilocytic astrocytoma in adults. However, bevacizumab, a monoclonal antibody against vascular endothelial growth factor, has shown to be beneficial in case series. Author Hayashi reports on the course of their patient in the NeuroImage, who received radiation and oral temozolomide. He agrees that bevacizumab may be useful in patients with anaplastic pilocytic astrocytoma and microvascular proliferation.

Megan Alcauskas, MD, and Steven Galetta, MD

Neurology ${ }^{\circledR}$ 2018;90:1037. doi:10.1212/WNL.0000000000005594

\section{Reader response: Pilocytic astrocytoma with anaplasia arising from the optic chiasm in a very elderly patient}

Andrea Wasilewski (Rochester)

Neurology ${ }^{\circledR}$ 2018;90:1037. doi:10.1212/WNL.0000000000005596

As alluded to by the authors of this NeuroImage, ${ }^{1}$ few studies have investigated pilocytic astrocytoma (PA) in adults, given the rarity of these tumors. One study suggested anaplastic PAs have a more aggressive clinical course when compared to juvenile PA with a higher incidence of tumor recurrence (from 30\% to $42 \%$ ) and progression. ${ }^{2}$ Specifically, PA with anaplastic features are more aggressive and associated with decreased survival when compared with typical PA. ${ }^{3}$ No standard of care exists for treatment of PA, especially in the elderly. Anti-angiogenic treatment, such as bevacizumab (a humanized monoclonal antibody directed against vascular endothelial growth factor), should be considered in patients with recurrent or unresectable PA. The highly vascular nature of these tumors suggests they may be uniquely responsive to bevacizumab and may have a similar response as seen in glioblastoma, including decreased permeability of vasculature and decreased perilesional edema. ${ }^{4}$ Case series demonstrated clinical and radiographic improvement as well as disease control with the use of bevacizumab. ${ }^{5}$

1. Hayashi S, Akao N, Nakazato Y, Okamoto K. Pilocytic astrocytoma with anaplasia arising from the optic chiasm in a very elderly patient. Neurology 2017;89:1840.

2. Ellis JA, Waziri A, Balmaceda C, et al. Rapid recurrence and malignant transformation of pilocytic astrocytoma in adult patients. J Neurooncol 2009;95:377-382.

3. Rodriguez F, Scheithauer BW, Burger PC, Jenkins S, Giannini C. Anaplasia in pilocytic astrocytoma predicts aggressive behavior. Am J Surg Pathol 2010;34:147-160.

4. Gilbert MR, Dignam JJ, Armstrong TS, et al. A randomized trial of bevacizumab for newly diagnosed glioblastoma. N Engl J Med 2014; 370:699-708.

5. Green R, Woyshner E, Quan J, Pope W, Cloughesy T. Treatment of unresectable adult pilocytic astrocytoma with bevacizumab with or without temozolomide (P01.097). Neurology 2013;80:P01.097.

Copyright @ 2018 American Academy of Neurology 


\title{
Author response: Pilocytic astrocytoma with anaplasia arising from the optic chiasm in a very elderly patient
}

\author{
Shintaro Hayashi (Sawatari, Japan) \\ Neurology ${ }^{\circledR}$ 2018;90:1038. doi:10.1212/WNL.0000000000005598
}

I thank Dr. Wasilewski for the valuable comments on our NeuroImage. ${ }^{1}$ Our patient received irradiation (57.6 Gy/32 fractions) and oral temozolomide (100 mg for $5 \mathrm{~d} / \mathrm{mo}$ for 14 months); however, the tumor gradually extended to the hypothalamus and he was transferred to another hospital for terminal care. We agree with Dr. Wasilewski's comment that alternative regimens for adult patients with anaplastic pilocytic astrocytoma (APA), especially showing recurrent or aggressive nature, are to be explored and established, and antiangiogenic treatment is one of the powerful tools. In our view, alternative treatments, such as bevacizumab, might be justified only when patients with APA show microvascular proliferations, as observed in our patient. ${ }^{1}$ We are not fully confident whether tests for BRAF V600E mutant proteins are mandatory, because the gene mutations are found in $6 \%-8 \%$ of extracerebellar pilocytic astrocytomas, ${ }^{2}$ as well as in other disorders (e.g., pleomorphic xanthoastrocytoma or ganglioglioma). ${ }^{3}$

1. Hayashi S, Akao N, Nakazato Y, Okamoto K. Pilocytic astrocytoma with anaplasia arising from the optic chiasm in a very elderly patient. Neurology 2017;89:1840.

2. Tanboon J, Williams EA, Louis DN. The diagnostic use of immunohistochemical surrogates for signature molecular genetic alterations in gliomas. J Neuropathol Exp Neurol 2016;75:4-18.

3. Shindler G, Capper D, Meyer J, et al. Analysis of BRAF V600E mutation in 1,320 nervous system tumors reveals high mutation frequencies in pleomorphic xanthoastrocytoma, ganglioglioma and extra-cerebellar pilocytic astrocytoma. Acta Neuropathol 2011;121: 397-405.

Copyright (c) 2018 American Academy of Neurology

\section{Editors' note: ELAPSS score for prediction of risk of growth of unruptured intracranial aneurysms}

In "ELAPSS score for prediction of risk of growth of unruptured intracranial aneurysms," the authors established a 6-predictor risk score for aneurysm growth. In their study, they defined significant aneurysm growth as greater than $1 \mathrm{~mm}$. Dr. Willey discusses the possibility of measurement error and bias as a result of this methodology. He also questions whether the readers were blinded, how aneurysm ruptures were treated statistically, and how ELAPSS compares to other existing classification systems. The authors did not respond.

Megan Alcauskas, MD, and Steven Galetta, MD

Neurology ${ }^{\circledR}$ 2018;90:1038. doi:10.1212/WNL.0000000000005599

\section{Reader response: ELAPSS score for prediction of risk of growth of unruptured intracranial aneurysms}

Joshua Z. Willey (New York)

Neurology ${ }^{\circledR}$ 2018;90:1038-1039. doi:10.1212/WNL.0000000000005595

In the article by Backes et al., ${ }^{1}$ the primary outcome was defined as greater than $1 \mathrm{~mm}$ growth as measured at local sites. The use of this cutoff was not referenced and a $1 \mathrm{~mm}$ change could be subject to potential measurement error. Furthermore, if different imaging modalities in one patient (magnetic resonance angiography vs CT angiography) were used to measure growth, 
their results could also lead to measurement error. An additional source of error and bias is if the physician carrying out the measurement was aware of the prior size. In that instance, the interpreting physician may judge an aneurysm that is larger to be more likely to grow, thereby biasing measurement. The potential sources of measurement error and bias may affect the results; it would be helpful to know if there was a blinded central reading center.

In the analysis, it was also unclear how aneurysm rupture was treated statistically. It appears that a stable aneurysm in 2 scans before rupture was treated as not having grown. Would this patient be treated as censored in survival analysis and not contribute information on aneurysm growth? Aneurysm rupture is a clinical outcome indicating a change in the aneurysm more serious than a $1 \mathrm{~mm}$ growth and with similar risk factors. The estimates, which had a large standard error, were heavily weighted toward size and location, which are well-known risk factors. To interpret the clinical and additive utility of ELAPSS, a statistical comparison to a traditional score with receiver operating characteristics or net reclassification would help. In other words, how much better was patient risk stratified with ELAPSS compared to a simple classification (e.g., posterior circulation and size greater than $7 \mathrm{~mm}$ )?

1. Backes D, Rinkel GJE, Greving JP, et al. ELAPSS score for prediction of risk of growth of unruptured intracranial aneurysms. Neurology 2017;88:1600-1606.

Copyright @ 2018 American Academy of Neurology

\section{CORRECTION}

\section{Multiparametric MRI changes persist beyond recovery in concussed adolescent hockey players}

Neurology ${ }^{\circledR}$ 2017;90:1039. doi:10.1212/WNL.0000000000004909

In the data supplement for article "Multiparametric MRI changes persist beyond recovery in concussed adolescent hockey players" by K.Y. Manning et al., ${ }^{1}$ there is an error in the "MRI Acquisition" section on page 4. The first sentence should read "All MRI data were acquired using a 32-channel human head coil" rather than "64-channel coil" as originally published. The authors regret the error.

\section{Reference}

1. Manning KY, Schranz A, Bartha R, et al. Multiparametric MRI changes persist beyond recovery in concussed adolescent hockey players. Neurology 2017;89:2157-2166. 